\title{
Recommendations for Laboratory Containment and Management of Gene Drive Systems in Arthropods
}

\author{
Mark Q. Benedict, ${ }^{1}$ Austin Burt, ${ }^{2}$ Margareth L. Capurro,, ${ }^{3,4}$ Paul De Barro, ${ }^{5}$ Alfred M. Handler, \\ Keith R. Hayes, John M. Marshall, Walter J. Tabachnick, and Zach N. Adelman ${ }^{10}$
}

\begin{abstract}
Versatile molecular tools for creating driving transgenes and other invasive genetic factors present regulatory, ethical, and environmental challenges that should be addressed to ensure their safe use. In this article, we discuss driving transgenes and invasive genetic factors that can potentially spread after their introduction into a small proportion of individuals in a population. The potential of invasive genetic factors to increase their number in natural populations presents challenges that require additional safety measures not provided by previous recommendations regarding accidental release of arthropods. In addition to providing physical containment, invasive genetic factors require greater attention to strain management, including their distribution and identity confirmation. In this study, we focus on insects containing such factors with recommendations for investigators who are creating them, institutional biosafety committees charged with ensuring safety, funding agencies providing support, those managing insectaries handling these materials who are responsible for containment, and other persons who will be receiving insects—-transgenic or not-from these facilities. We give specific examples of efforts to modify mosquitoes for mosquito-borne disease control, but similar considerations are relevant to other arthropods that are important to human health, the environment, and agriculture.
\end{abstract}

Keywords: gene drive, biosafety, transgenic organism, mosquitoes

\section{Introduction}

D RIVING TRANSGENES ARE SYNTHETIC GENES that have the capacity to spread in natural populations even if they confer a fitness cost. Driving transgenes have been identified as candidates for control of pests and disease (Burt 2003, Sinkins and Gould 2006, Deredec et al. 2011, Gabrieli et al. 2014, Webber et al. 2015) and include nuclease-based gene drive systems (Gantz et al. 2015, Hammond et al. 2016); "Y-drive" for population suppression (Bernardini et al. 2014, Galizi et al. 2014); Medea, including synthetic vari- ants, for population replacement (Hay et al. 2010, Ward et al. 2010); and class II transposable elements (Kidwell and Ribeiro 1992). The biology, risks, and uses of the most recently devised CRISPR/Cas9 system have been highlighted (Esvelt et al. 2014, Webber et al. 2015), although not all invasive factors have attracted as much attention. A variety of available drive systems, including transgenic, natural, and chromosome rearrangements, is reviewed elsewhere (Burt 2014, Marshall and Akbari 2016).

These driving factors have the potential to invade target populations of a particular species after introduction of

\footnotetext{
${ }^{1}$ Entomology Branch, Centers for Disease Control and Prevention (CDC), Atlanta, Georgia.

${ }^{2}$ Life Sciences, Imperial College London, Ascot, United Kingdom.

${ }^{3}$ Department of Parasitology, Institute of Biomedical Sciences, University of Sao Paulo, Sao Paulo, Brazil.

${ }^{4}$ National Institute of Science and Technology in Molecular Entomology, National Council of Scientific and Technological Development (INCT-EM/CNPq), Rio de Janeiro, Brazil.

${ }^{5}$ CSIRO, Brisbane, Australia.

${ }^{6}$ USDA-ARS, Center for Medical, Agricultural, and Veterinary Entomology, Gainesville, Florida.

${ }^{7}$ CSIRO, Hobart, Australia.

${ }^{8}$ Divisions of Biostatistics and Epidemiology, School of Public Health, University of California, Berkeley, California.

${ }^{9}$ Florida Medical Entomology Laboratory, Department of Entomology and Nematology, University of Florida, Vero Beach, Florida.

${ }^{10}$ Department of Entomology, Texas A\&M University, College Station, Texas.

(C) Mark Q. Benedict et al. 2018; Published by Mary Ann Liebert, Inc. This is an Open Access article distributed under the terms of the Creative Commons Attribution License, which permits unrestricted use, distribution, and reproduction in any medium, provided the original work is properly cited.
} 
individuals carrying the factor that we refer to here as an invasive genetic factor (IGF). The characteristics that make them attractive for development also require consideration of the risks that are unique to the technology. Their potential for spread is a unique characteristic that adds an added layer of complexity to case-by-case risk assessment. IGF technology in general may therefore require different safety considerations to prevent unintended release than the considerations that have been applied for other arthropods since once released, some IGFs are intended to spread throughout a population. In addition to the possible environmental concerns, such releases could have legal and ethical implications relevant to interstate and international movement or transmission. Therefore, because movements and transfers of genetically modified organisms are covered under specific national laws and international agreements that do not cover nonmodified organisms, they warrant special consideration that we will describe in more detail.

The potential application of IGFs is a variation of genetic control that has been defined as "reducing pest damage using factors that disseminate by mating or inheritance" (Benedict and Scott 2016). Unlike traditional sterile insect technique (SIT) control methods that usually involve the sustained inundative release of irradiated sterile males, IGFs are not expected to require repeated releases with large numbers of insects to accomplish a beneficial effect. Rather, they have the potential to spread naturally following inoculative releases of a small number of individuals. This could reduce costs greatly because release programs of a limited scale and duration can be envisioned to result in effective and durable control.

This article focuses on IGFs and is directed toward developers who are creating strains containing an IGF, managers of insectaries containing these materials, those who will be receiving insects from these facilities, Institutional Biosafety Committees (IBCs) charged with approving the safety procedures in place to ensure containment, granting agencies, and review panels. We describe the measures in various existing forms of containment guidance and explain that while these provide a useful basis for containment of arthropods containing IGFs, they leave a deficit in the measures for distinguishing and managing such strains. If legal guidance is formulated for containment of IGFs in laboratory settings, these considerations may shape their content; "...such guidelines and standards can have a formative role in the early development of radically innovative technologies to guide future regulatory decisions." (NAS Committee 2016). We focus on low-threshold driving transgenes and other IGFs that can spread autonomously and do not discuss in detail binary systems, in which the molecular components occur in parts that are not linked genetically and cannot spread independently. Such binary systems have been proposed as a means to prevent inadvertent spread that can be used experimentally, but do not appear to be as useful for release purposes as low-threshold autonomous systems (Akbari et al. 2015). We primarily use mosquito transgenesis examples because we have experience in this field, but we believe the principles will serve as normative principles relevant to other organisms. While we make recommendations for managing and containing strains carrying IGFs, the recommendations are intended to provide a basis for formulating procedures to ensure safety and prevent accidental release, thereby assisting approving authorities in conducting appropriate risk assessments.

\section{Potential of Invasive Genetic Factors}

\section{Spread of an IGF can be beneficial}

IGFs are being developed to supplement inadequate approaches to controlling pests and, in some cases, the diseases they transmit. Recent advances in malaria control (WHO 2015), while successful at significantly reducing the burden in sub-Saharan Africa (Bhatt et al. 2015), leave gaps for which existing control tools will be inadequate to reach elimination in high prevalence areas (Griffin et al. 2010). Similarly, mosquito-borne viruses pose an increasing worldwide threat that is not adequately controlled by vector control or vaccines. Mosquito control is an ongoing need even when diseases are not being transmitted in an area. For example, arboviruses can emerge unexpectedly as demonstrated by the rise of Zika (Weaver et al. 2016) and chikungunya (Cao-Lormeau 2016). Among other approaches, IGFs are being developed as candidates to meet this need. They potentially also have the capacity to suppress populations of medically important invasive species such as Aedes albopictus.

Furthermore, it is likely that IGFs for controlling agricultural pests will be explored (M. Scott, pers. comm.). For example, only intensive insecticide application has had any impact in reducing populations of the Asian citrus psyllid, which is responsible for transmission of the pathogen causing citrus greening, devastating the U.S. citrus industry (GraftonCardwell et al. 2013). Currently, there are no adequate control methods available for this insect. While other pests can be controlled with insecticides, IGFs may offer a less costly and more environmentally sound control method. The commercial benefit and potential for low-cost implementation will make these attractive research subjects. Thus, issues of insectary containment of IGFs as well as controlled evaluations will be an expanding concern that should be addressed early in their development.

\section{Unwanted spread of an IGF in the environment}

By design, some driving transgenes have the potential to spread widely and rapidly from a small introduction and could result in extensive genotypic and/or phenotypic changes in the targeted pest (Marshall 2009, North et al. 2013), potentially with effects beyond the primary intent (Tabachnick 2003). From the initial stages of laboratory research, those working with IGFs should be cognizant of potential consequences in resulting phenotypes, including the possibility of unanticipated pleiotropic effects in an unknown and uncharacterized genetic background and the environmental context of variation in naturally occurring target pest populations. The considerations that we present here are specifically designed to prevent the accidental premature release of a transgene before it has been evaluated for potential effects on the target organism's ecological relationships and the environment and the release has been approved by authorities who are responsible for public and environmental safety.

\section{Invasive Genetic Factors and Systems}

While genetic control of arthropod vectors generally has been recognized by the $\mathrm{WHO}$ as a promising technique against mosquito-transmitted diseases (WHO/TDR 2010), each IGF and arthropod system will have unique characteristics that must be considered on a case-by-case basis. Case-by-case 
assessments should address the intended use and design of the IGF, potential risks of the IGF strategy, appropriate containment, and utility for population replacement or suppression.

Some IGFs are predicted by models to have a low population frequency threshold that must be exceeded to invade, hence releases of only a few individuals (such as might occur as a result of containment failure) could theoretically result in invasion (Marshall 2009). Arthropods containing these potentially low-threshold IGFs may warrant higher containment stringency and management procedures than those applied to arthropods containing high-threshold IGFs or noninvasive genetic modifications. IGFs that act through homing-based mechanisms such as homing endonuclease genes (HEGs, Windbichler et al. 2011) and CRISPR (Gantz et al. 2015) may display strong gene drive even at low introduction rates. Under neutral fitness conditions and without containment measures, models predict that these systems could persist even for release sizes as small as five individuals (Marshall 2009). An increase in the prevalence of a transgene in a population can result in broadening spatial distribution of the IGF. Increasing prevalence and distribution of driving transgenes, however, is not certain and depends upon many factors, including the fitness conferred on individuals carrying the IGF, the biological permissiveness of the season when release occurs, and the drive strength (Eckhoff 2017). Other systems such as Medea (Hay et al. 2010), when used for population replacement, display a drive strength that depends on their initial abundance relative to the existing population, with the strength of drive being weak at low population frequencies and high at intermediate values. Small numbers of escapees carrying these systems are likely to be lost through genetic drift (Marshall 2009); however, larger accidental releases may result in spread, and local population structure may assist this.

Underdominance-based approaches are unlikely to spread subsequent to small accidental releases (Marshall 2009) and are likely confinable to isolated populations and could be eliminated through releases of wild-type individuals (Marshall and Hay 2012). An example is the $\mathrm{UD}^{\mathrm{MEL}}$ system engineered by Akbari et al. (2014). These systems display threshold properties such that a superthreshold release is required for spread, and this is unlikely to be achieved through migration to neighboring populations. We anticipate that containment and management requirements for these strains will not need to be as stringent as for IGFs without such high thresholds.

\section{Invasive Genetic Factors Present Novel Consequences to Consider}

When present at low frequencies in populations, alleles and noninvasive (i.e., conventional) transgenes can be lost due to a variety of reasons, including genetic drift or reduced fitness (Fisher 1922, Haldane 1927), unless the trait conferred is under positive selection. In contrast, the increase in frequency of driving transgenes and other IGFs in populations without conferring any specific selection advantage means that they-as a class of transgenes — can potentially increase in frequency over time and spread spatially through preferential inheritance and population dispersal and migration. This presents a novel situation that those who are responsible for conducting research with arthropods safely may be unfamiliar with.
To illustrate how an accidental release resulting in establishment of the transgene in wild populations in the vicinity might occur, we describe two scenarios:

The first example considers a strain of arthropods containing only one IGF being maintained under existing containment measures for transgenic arthropods (ACL-2), as defined in the Arthropod Containment Guidelines (ACGs, ACME/ASTMH 2003). Briefly, ACL-2 recommends sealed ventilation and plumbing penetrations, vestibules, isolated spaces for work with transgenic organisms, devitalization before disposal, restricted access, and shares features of other guidance listed in Table 1. These guidelines were developed by the American Committee for Medical Entomology (ACME), a subgroup of the American Society of Tropical Medicine and Hygiene. The ACGs have no legal authority, but are often used, particularly in the United States, in describing appropriate measures for containment of arthropods not covered elsewhere (see below regarding U.S. guidance). Containment failure due to facility shortcomings, human error, or other reasons allows a fertile individual to escape into a wild population near the facility, resulting in predominance of the IGF in the wild population. In the context of this discussion, we stress that the probability of a driving transgene becoming established in wild populations is much greater than that of a non-IGF.

In a second scenario, a wild-type (nontransgenic) strain is being handled in a laboratory that also handles the same species or a species that mates compatibly, but containing an IGF, both strains being under ACL-2 containment. This situation is common because many laboratories that create transgenic insects maintain nontransgenic strains as phenotypic references in the form of wild-type comparators or as the host strain. According to most guidance (described further below), both should be maintained at the highest containment level that applies to either. Unbeknownst to the insectary manager, contamination of the wild-type strain has recently occurred due to accidental transfer of individuals from the IGF-containing strain. The wild-type strain is presumed to be free of any IGF and is shipped to a collaborating laboratory where it is handled under ACL-1 containment

Table 1. Measures Shared by Arthropod Containment Guidelines That Are Appropriate FOR InVASIVE Genetic Factor Arthropods

Activity isolation to prevent accidental and unauthorized intrusion

Restricted access, often with staff training required

Sealed penetrations, including ventilation, sewage, windows, and doors

Multiple partitions between primary containment and the outside

A limited number of entrances with either a vestibule or airlock and sometimes both

Controlled directional air flow

Smooth pale walls and floors suitable for disinfesting and detecting escapees

Secure primary containment

Devitalization of arthropods before disposal

Inspection and disinfestation of equipment and materials before removal from containment

Autoclave accessible or in containment suite

Lab coats and gowns when pathogens present 
measures that are appropriate for wild-type strains in locations where the same species occurs naturally. Due to the lower level of containment measures in the receiving laboratory compared with the shipping laboratory, an accidental escape from the laboratory results. Similarly to the first case, release of the IGF results in invasion of the wild population.

It follows that not only must laboratories working with low-threshold IGFs act appropriately to prevent escapes and introduction into non-IGF strains within the laboratory but also that the strains handled within the insectary must be carefully managed before shipment to other laboratories to ensure that an IGF is not distributed.

These two scenarios illustrate the questions investigators and those charged with ensuring safe handling of arthropods with IGFs can be expected to answer. Can one be certain that a strain being held in a laboratory does not contain an IGF? Can one be certain that those that are known to contain an IGF do not contain more than one type? If non-IGF strains that are being maintained in laboratories that handle IGFs will be distributed or exchanged among other laboratories, can assurance be provided to recipients that the strains do not contain IGFs? Should the recipient be able to verify this? How stringent does detection need to be?

\section{Existing Arthropod Containment Guidance}

Arthropod containment guidance for compliance with specific standards differs among countries according to institutional, funder, or national regulation and guidance. These are typically shaped by various considerations, including risk assessments, historical experiences, and political and cultural influences. Some countries have no national guidance and refer to procedures of other countries or nongovernmental organizations such as the ACGs of ACME. To make the considerations presented here applicable broadly, we surveyed several standards for arthropod containment to determine the specific measures they share and those that distinguish them (Supplementary Table S1; Supplementary Data are available online at www.liebertpub.com/vbz). We also considered whether specific measures that are recommended are needed primarily to contain a pathogen or the arthropod.

A survey of several relevant English language containment guidance documents shows that these recommendations are remarkably similar (Supplementary Table S1). We considered the Australian/New Zealand standards; PC2 (www.ogtr .gov.au/internet/ogtr/publishing.nsf/content/PC2-4/\$FILE/ PC2LABv3-1-1.pdf), PC3 (http://ogtr.gov.au/internet/ogtr/ publishing.nsf/Content/PC3-4/\$FILE/PC3LABv3-May2012 .pdf), and the QIC2 (www.agriculture.gov.au/import/arrival/ arrangements/qap/class7/qap-criteria-72/21_scope) and QIC3 (www.agriculture.gov.au/SiteCollectionDocuments/biosecurity/ import/general-info/qap/class7/class-7.3.pdf) quarantine standards, the UK SAPO 8000 standards for arthropods (www .gov.uk/government/uploads/system/uploads/attachment_data/ file/400360/animal-pathogens-guidance-controls.pdf), and the Canadian PPC2 and PPC3 (www.inspection.gc.ca/plants/ plant-pests-invasive-species/biocontainment/containmentstandards/eng/1412353866032/1412354048442?chap=4\#s14c4) standards. We also examined the USDA-APHIS-PPQ guidance for Nonindigenous, Phytophagous Arthropods and Their Parasitoids and Predators (www.aphis.usda.gov/plant_health/ permits/downloads/arthropod_biocontrol_containment_guide lines.pdf) and the nongovernmental Arthropod Containment Guidelines (ACME/ASTMH 2003) that address both transgenic, infected, and uninfected exotic and native species.

There is a remarkable parallel even in the nomenclature across standards presented in each document. Level 2 is not stringent for airborne pathogen containment, whereas level 3 adds measures, including more closely controlled air pressures, sewage disinfection or sterilization, access restriction, air management and filtration (including HEPA), and activity isolation. Therefore, we will refer to level 2 and level 3 containment as a reflection of this broad consensus among standards that distinguish those levels. There are, in fact, a limited number of ways to contain arthropods (Table 1), and approaches for doing so are found in most of the guidance documents reviewed. There are essentially three ways that accidental escape can occur: (1) any living stage, including immatures in solid and liquid waste; (2) transport on equipment or staff; and (3) penetrations of the containment zones, including doors, windows, and ventilation. Escapes by all of these means are avoidable, and preventive measures are the goal of the various standards. The same principles apply whether the arthropod is exotic, a potential biocontrol agent, a vector of plant and human diseases, or transgenic. These measures are effective for confining arthropods.

As containment stringency increases above level 2, the added layers are generally those measures needed to contain a pathogen in addition to those for the arthropod. This often results in escalating measures specifically needed to effectively contain a higher risk of particularly airborne pathogens, but which have no clear benefit for containing the arthropod. Figure 1 illustrates the overlap that exists between arthropod and microbiological containment measures as manifested in containment standards.

We recommend that in the case of arthropods containing low-threshold IGFs in the absence of any pathogen, any increasingly stringent measures should include those that effectively contain arthropods rather than those that are designed to contain pathogens. We describe several possibilities for doing so in the next section.

All levels of the arthropod containment measures reviewed require good primary containment (caging), sealed walls, openings, and doors, usually vestibules or airlocks, surfaces that can be disinfected (or disinfested), appropriate personal protection equipment, and controlled access (Table 1). Only one of these allows windows that can be opened (PC2). All require devitalization of arthropods before disposal (although the means differ) and some require liquid effluent to be sterilized. Air curtains were rarely recommended and negative air pressure was consistently emphasized at higher containment levels, although often still recommended as a gradient without specification of the value of the pressures at level 2. While most standards do not explicitly require segregated working spaces, they specify measures such as vestibules and restricted access that would be difficult to achieve unless activities were confined to segregated spaces. Overall, containment guidance documents surveyed described similar requirements with one interesting exception: PC3 requires that provision must be made for viewing of work areas from outside the facility. The commonalities across these standards demonstrate that methods for containing arthropods are consistent and feasible. 


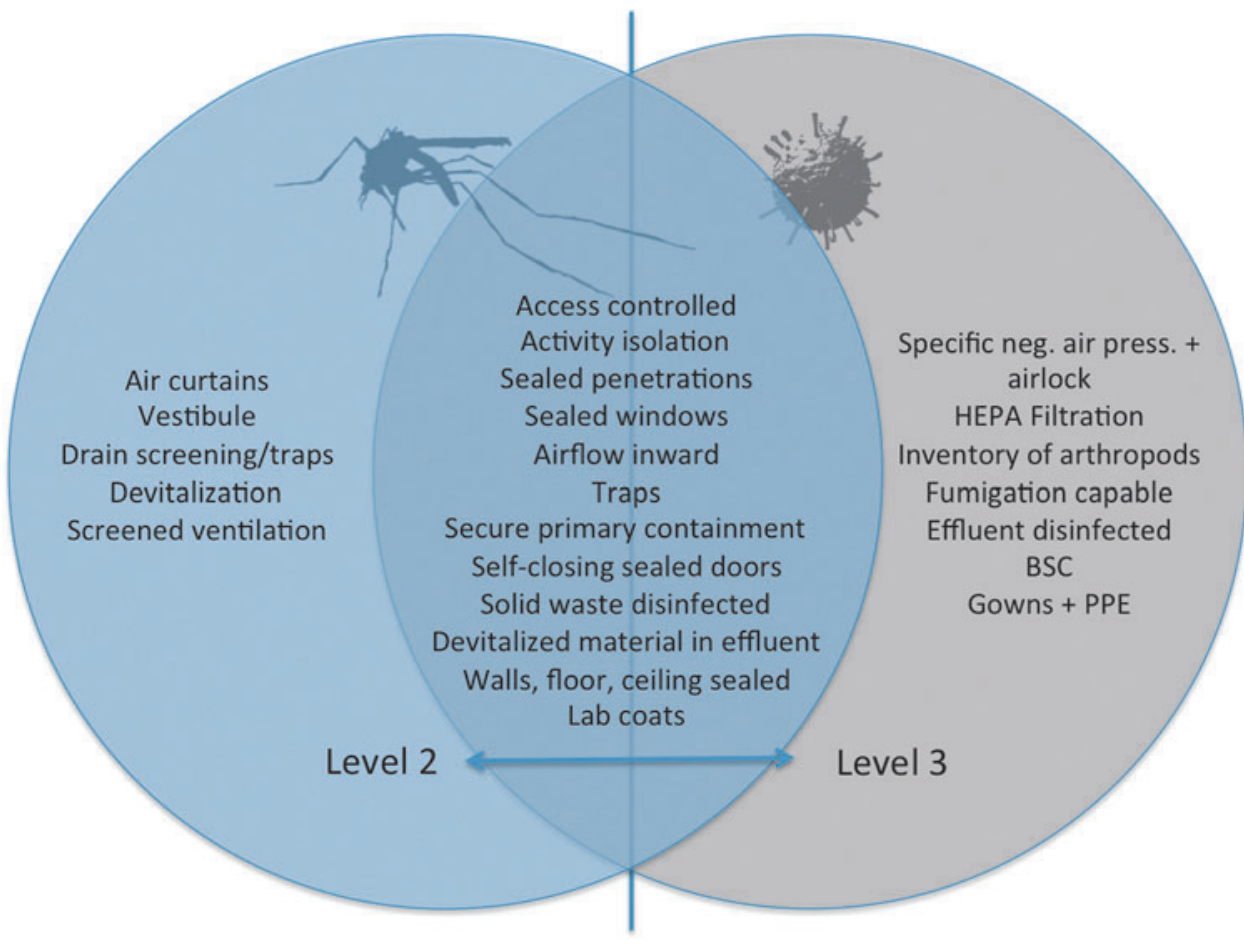

FIG. 1. The overlapping measures to contain arthropods and microbes in facilities handling arthropods. Measures that will contain a microbe will not necessarily contain an arthropod, and vice versa. The arthropod containment measures (left) overlap with those specific for microbes (right). The stringency of measures in the overlapping area depends on whether the facility is level 2 or level 3. In the case of IGFs, the goal is to contain arthropods containing the factor and the use of stringent microbiological methods adds no effect. IGF, invasive genetic factor.
The issue of segregation of materials that require different levels of containment is specifically addressed in many standards for level 3 facilities. These specify that lower-level materials in the same facilities must be handled as higherlevel materials. This is an important issue that we will return to below.

\section{The Patchwork of Guidance in the United States}

\section{NIH guidelines}

Oversight for the generation and/or use of recombinant DNA-containing organisms in research institutions is provided by the NIH Guidelines and is, in turn, delegated to individual institutions for enforcement. The insertion of recombinant DNA into any arthropod currently falls under section III-D-4 of the NIH Guidelines for Research Involving Recombinant or Synthetic Nucleic Acid Molecules (http:// osp.od.nih.gov/sites/default/files/NIH_Guidelines.html), which we refer to simply as the NIH Guidelines, and thus requires approval by an IBC before initiation. Of note, IBC approval is required irrespective of whether the modified strain is made locally or made at another institution and imported. In the absence of any microorganisms that might warrant a higher level of containment, the genetic manipulation of arthropods falls into NIH Guidelines, subsection III-D-4-a, with recommended containment of BL1 as specified in Biosafety in Microbiological and Biomedical Laboratories (BMBL, Department Health and Human Services, 2016). BL-1 is a standard established for containment of certain microorganisms.

In some cases, local IBCs may utilize the recommendations laid out in the ACGs and recommend containment at ACL-2 (or beyond), but this may not always be the case. ACL-2 was deliberately specified to be consistent with BL-2 containment. If BL2 containment is applied, section III-G-B$2-\mathrm{k}$ specifies that spills and accidents that result in overt exposures to organisms containing recombinant or synthetic nucleic acid molecules are immediately reported to the IBC and NIH/OBA; no such requirement is made at BL-1. In practice, it is not clear whether the bite of a recombinant DNAcontaining arthropod presents the same threat to laboratory workers as infection with a recombinant DNA-containing microorganism. The NIH Guidelines also specify physical containment conditions that represent best practices for laboratory containment (Appendix G of the NIH Guidelines), although these practices are written in terms of protecting laboratory workers and the environment from pathogenic microorganisms, and do not make any specific recommendations in terms of requirements for the effective physical containment of arthropods.

However, some useful arthropod containment suggestions can be gleaned from descriptions of physical containment for plants in a greenhouse setting that may supersede the conditions described in Appendix G (Appendix P of the NIH Guidelines). For example, similar to IGFs used in arthropods, the primary goal of containment laid out in Appendix $\mathrm{P}$ is to minimize or prevent "...the unintentional introduction and establishment of an organism in a new ecosystem" (section P-I-B). Appendix P specifies directly that a record shall be kept of experiments currently in progress in the greenhouse facility [section P-II-A-1-b-(1), P-II-B-1-b-(2), P-II-B-1-b(2), and P-II-D-1-b-(2)], an important consideration for arthropods as well. For plant containment levels 2-4, another sound requirement that would apply equally well to insectaries handling IGFs is "A record shall be kept of experimental plants, microorganisms, or small animals that are brought into or removed from the greenhouse facility" [P-IIB-1-b-(1)]. Good record keeping of shipments received and 
made to any facility handling transgenic arthropods is essential. Ultimately, the NIH guidelines (Appendix Q-III-D) defer on specific recommendations for nonlaboratory animals such as arthropods, settling for any combination of containment conditions that meets the satisfaction and approval of the local IBC.

Laboratories in the United States that apply for a United States Department of Agriculture (USDA) Biotechnology Regulatory Services permit to transfer or import certain classes of organisms, including transgenic insects, are subject to inspection and approval for a USDA Veterinary Services permit. The containment criteria to which receiving facilities are subject to are a combination of the BMBL level 2 recommendations and arthropod-specific measures similar to those in the USDA-APHIS-PPQ guidance described above.

The Centers for Disease Control and Prevention (CDC) provides a practical checklist of features required for ACL-2 approval to obtain a US Public Health Services permit for arthropod vectors relevant to human health (www.cdc.gov/ od/eaipp/inspection/docs/import_permit_checklist_acl-2 .pdf). The features are similar to those prescribed by other guidance documents and are not specifically itemized here.

\section{Arthropod containment guidelines}

In part, to fill gaps in the practical measures of the NIH Guidelines in the United States, the Arthropod Containment Guidelines were created to recommend containment levels specifically for arthropods (Arthropod Containment Levels 1-4). These recommend ACL-2 for the containment of transgenic arthropods. Although these guidelines have been used widely in the United States and have been incorporated into several national containment standards, they did not anticipate the unique hazards posed by IGFs. Containment and testing measures for driving transgenes in field cages have been discussed previously (Benedict et al. 2008).

\section{Considerations That Affect Risk and Physical Containment Decisions}

It is important when assessing risks, particularly from hypothetical or speculative hazards generated by what-if scenarios, to keep in perspective the risks presented by the unmodified wild-type comparator. For vector control strategies, the risks associated with the IGF-containing arthropod need to be balanced against the disease burden that the unmodified arthropod poses even with optimal use of the existing conventional control methods, including insecticides. For example, two driving transgene strains of malaria vector mosquitoes, An. stephensi and An. gambiae, were recently created, one containing single-chain antibodies against malaria ookinetes and sporozoites (Gantz et al. 2015) and the other reducing the reproductive potential of female mosquito (Hammond et al. 2016). Both of these strains were created to diminish the harm that is known to be caused by a wild-type comparator, and each could be considered to present less risk in the event of escape (in the event of establishment) than the wild-type comparator, that is, their possible risks need to be balanced against the known harms of the wild type.

More study of the modified mosquito phenotypes, for example, effects on range, host preference, or biting activity that might present risks, is required beyond the initial investigations that are focused on the trait that has been de- liberately modified. This initial uncertainty dictates that while the primary phenotype is important in considering risk, continued caution must be exercised until these other questions are answered during the development of the arthropod. This is one of the reasons why a progressive phased evaluation of transgenic arthropods is recommended during which their performance against model predictions, environmental interactions, and health risks is evaluated (WHO/FNIH 2014, NAS Committee, 2016). Such a process permits the collection of risk-related data that best predict their behavior and upon which a review of appropriate containment for the next level of development can be based.

\section{Ecological Containment}

\section{Where the environment is not suitable}

Ecological (also called environmental) containment exists when activities are being conducted in a location where, in the event of escape, the arthropod would not become established due to an unfavorable environment. With regard to the IGF rather than the arthropod, this situation is analogous to the containment that exists as the result of the absence of an existing population in the vicinity of the insectary into which the IGF can propagate, but with the additional safeguard that probability of an escape population becoming extinct is high due to an unsuitable environment. This approach was explicitly recognized in the ACGs and is at least implicitly recognized by most other guidance documents described above. The expected benefits of ecological containment for species containing drive systems were again asserted as a reasonable measure in recent published reports of discussions of drive system containment (Esvelt et al. 2014, Oye et al. 2014, NAS Committee 2016) assuming that the IGF does not expand the species' environmental niche. In general, however, it is reasonable to expect that the degree of physical containment demanded under these conditions would reflect the relatively much lower probability of IGF persistence in the environment, by virtue of the protection afforded by the ecological containment, and the concomitant reduction in risks that result.

\section{Where the environment is suitable}

There are other locations where the arthropod does not occur, but where the environment is ecologically suitable for establishment. This presents the possibility of persistence of the IGF in the environment in the newly established population that resulted from escapees. Again, this must be assessed on a case-by-case basis, where the degree of physical containment required would be informed by the phenotype conferred by the IGF, probability of escape, potential size of the escaping population (Hayes and Barry 2008), and subsequent risks if establishment occurs. Expansion of the distribution of the arthropod following an escape could result in mating with a nearby wild population or a geographically distant population. This could pose less immediate, but similar, risks to the situation described next. For both situations, the protection afforded by ecological containment is diminished; hence, precaution against accidental release through additional arthropod-specific containment is required and will be described further below. 


\section{Where the arthropod is present}

When the species occurs at the location of the laboratory, there is a much higher risk of spread if the IGF escapes. In addition to stringent physical measures to prevent escape, efforts should be made to utilize the most effective trapping methods to monitor both inside and outside of the facility to verify with some certainty that escapes are not occurring. The creation of ecological buffer zones outside the facility (devoid of breeding sites, resting locations, or hosts) may be warranted. Regardless of whether the trait conferred by the IGF is believed to be beneficial or not, the unintended and uncontrolled nature of its invasion would make efforts to interfere with its spread difficult. In this case, the strictest arthropod containment measures are recommended.

We cannot stress too strongly that such measures should be focused on arthropod containment rather than merely assignment of a more stringent level developed to contain microorganisms. Advanced features such as highly controlled airflow and centralized waste sterilization and segregated mechanical spaces raise the cost of construction and operation greatly and may be necessary for containment of microbes, but without contributing significantly to containment of the arthropod. Costly and unnecessary requirements for containment measures that do not substantially increase arthropod containment could stifle efforts to build research capacity in disease-endemic and developing countries and may simply not be possible in many situations involving preparation and testing of an IGF-containing strain.

\section{Uncertainty Associated with the Norm of Reaction}

An important challenge in assessing the risk for IGFcontaining arthropods is the uncertainty regarding possible pleiotropic effects and our limited ability to characterize the norm of reaction of a modified gene. The norm of reaction refers to the expression of genotypes in relationship to a range of environments. This consideration becomes more important as arthropods that contain an IGF proceed from preliminary testing in the developing laboratory on to confined field testing. The capacity to measure these reactions also becomes more feasible and realistic during this progression; however, some effects can be measured early in this progression and may affect the containment decisions. For example, how does the phenotype vary at different temperatures that the transgenic insect will encounter or when exposed to natural pathogens? The ability to predict phenotypic consequences of inserted transgenes on vector competence for pathogens may be affected by uncharacterized naturally occurring genetic and environmental variations (Tabachnick 2003, 2013). Strains that are designed to debilitate or suppress a target species may also be affected by natural biological variation in the target populations and loss of these phenotypes under certain conditions may affect the risk conclusions.

This uncertainty may complicate risk assessment, and because of this, the assessment cannot be reduced by simply comparing the phenotype of the IGF strain observed under laboratory conditions with the phenotype of the wild-type comparator observed under natural conditions. This also is one of the reasons why previous guidance for genetic vector control technology (Benedict et al. 2008, WHO/FNIH 2014) recommends a phased test and release strategy wherein the modified arthropod is evaluated under a series of increasingly realistic conditions (that deliberately and gradually relax containment when warranted) before open release into the environment. The aim of confined field trials, for example, is to observe the phenotype of the genetically modified strain in a genetic background and under environmental conditions that are as close as possible to those that occur naturally in an attempt to detect undesirable pleiotropic effects that might not be evident under the artificial conditions of laboratory culture. Observations taken during the staged testing and release pathway that indicate that an IGF conferred a characteristic that made a harmful arthropod more hazardous than a wild-type comparator, for example, increased vector competence for the pathogen under study, or changed biology making it more difficult to control, could be grounds for terminating further development of the strain.

\section{International Legal Considerations}

Because IGFs have the theoretical potential to spread across international boundaries, which may eventually be desirable for providing sustained beneficial effects, both the intended and inadvertent release of an IGF in even a model species can have legal implications that should be taken into consideration. The Cartagena Protocol, for instance, applies to the "transboundary movement, transit, handling and use of all LMOs that may have adverse effects on the conservation... of biological diversity" (Secretariat of the Convention on Biological Diversity, 2011). Here, the term LMO is analogous to a genetically modified organism and, in the words of the Cartagena Protocol, refers to any living organism that possesses a novel combination of genetic material obtained through the use of modern biotechnology.

The possibility of legal redress and liability under this convention due to spatial spread should also be considered. Parties to the Cartagena Protocol are expected to take appropriate measures to prevent unintentional transboundary movements (Article 16 of the protocol). However, if such movements do occur, the protocol states that the country where the environmental release occurred shall immediately consult the affected or potentially affected States to enable them to determine appropriate responses and initiate necessary action, including emergency measures (Article 17 of the protocol). The Nagoya-Kuala Lumpur Supplementary Protocol on Liability and Redress (Secretariat of the Convention on Biological Diversity 2011) applies to damage resulting from transboundary movements of LMOs and includes movements originating from nonparties in its scope (Article 3 of the Supplementary Protocol). The Supplementary Protocol currently requires only nine more countries for ratification.

\section{Containment Above Level 2-“Level 2+”}

Because there is remarkable consensus of arthropodspecific recommendations across all of the guidance we have considered and most arthropod-specific measures are recommended at level 2, if level 2 were adopted (along with select level 3 arthropod measures), what more would, and would not, be needed to ensure containment? We recommend against simply escalating physical containment measures to the next higher level. For example, ACL-3 specifically advises practices suitable for work with potential or known vectors that are, or may be infected with, BSL-3 agents 
associated with human disease, but none of these characteristics necessarily exist for IGF-containing arthropods. Similarly, increasing the level of containment from QIC2 to QIC3 and the accompanying sterilization (rather than disinfection) of waste and requirement for HEPA air filtration provide little additional benefit for containing arthropods containing an IGF. In the following section, we discuss individual measures that could be considered to improve the containment above level 2.

\section{Airlocks, Negative Pressure, and Air Showers}

\section{Airlocks}

Airlocks, as implemented in insectaries, typically consist of an antechamber in which both doors are not opened simultaneously and which are capable of maintaining negative air pressure. Vestibules are similar spaces that do not maintain air pressure differentials. Both are sometimes equipped with air curtains, traps, and fans, which are often recommended. Insectaries handling IGFs should consider whether higher containment airlocks are needed and determine whether multiple doors and other measures are sufficient to isolate strains containing IGFs from other arthropod stocks and natural environments where the establishment of the wild type is possible. The accidental transfer of immatures through such airlocks is possible and routes of transfer on clothing, personnel, and in waste should be considered. Consideration of additional procedures that would prevent such transfer may be required such as tack-trap door guards, shower facilities, or dunk tanks for small crawling arthropods such as ticks as proposed for ACL-3 in the ACGs.

\section{Negative air pressure}

The general requirement for negative air pressure in an insectary relative to the outside environment is based on microbiological containment measures for which it is clearly justified since microorganisms can be dispersed in air streams (www.aphis.usda.gov/plant_health/permits/downloads/ arthropod_biocontrol_containment_guidelines.pdf). This was reiterated and mirrored in an attempt to make the ACGs consistent with BMBL guidelines and is often recommended particularly, but not exclusively, when microbes are in containment. Unlike microbes, arthropods crawl, fly, and hop. Negative pressure that is suitable for microbiological containment provides no protection against departure of any, but the weakest and lightest, arthropods and does nothing to prevent the accidental transport of immatures. For mosquitoes, negative pressure might actually increase the risk of escape since at least some species are stimulated to fly upwind in gentle $(0.2-0.5 \mathrm{~m} / \mathrm{s})$ air movement (Gillies and Wilkes 1981, Lefevre et al. 2009). Regardless, there is one advantage of negative pressure inside containment: it enables investigators to see even small holes in the containment facility envelope due to dirt carried by escaping air that accumulates around any holes. This can also be assessed by pressure tests or by filling rooms with artificial smoke as part of the commissioning process and searching for escaping streams that identify leaks. Penetrations could also be detected using similar methods in facilities that do not maintain negative pressure. The advisability of this approach to accomplish containment should be considered on a case-by-case basis.

\section{Air showers}

More stringent containment of an IGF over that provided by air locks would be separation of areas holding IGF strains by air showers rather than simple airlocks. These are used in cleanroom manufacturing and biocontainment facilities and provide strong, directional, and filtered air circulation in the chamber. The turbulence and force of air movement would prevent an adult arthropod such as a mosquito, biting midge, or psyllid from moving in a person's hair, a means of movement that we have observed previously (Tabachnick, Benedict, unpublished). However, air showers or strong airflow of any kind should not be used where the potential for aerosolization of infectious agents exists (e.g., virus-infected mosquitoes).

\section{Physical Containment Provided by Handling Methods}

Several methods for handling insects in primary containment (e.g., cages, vials of rearing medium) were recommended by Gantz and Bier (2015). These included anesthetizing flying forms in containers and counting them etc., similar to the measures that are used for handling infected arthropods in BL-3 facilities under ACL-3 containment. These are potentially useful and should be considered as one part of a containment system where they are compatible with the culture of the organism. Adults of small arthropods such as biting midges and psyllids are more difficult to count due to their small size. Immature forms such as arthropod eggs can pose as much, or indeed greater, risk as adult arthropods if accidentally released. It is difficult to count these stages and it can be far more difficult to visually identify their escape from a container.

Recommendations for wearing gloves during manipulations that do not involve pathogens should be considered carefully since they may interfere with sensitive handling of small arthropods.

\section{Markers for Detecting Transgenes}

Transgenic individuals are identified by a unique, usually visible, phenotype conferred by a marker on the transgene or more rarely by a unique phenotype created by interrupting a specific gene upon insertion. Phenotypic markers that would identify transgenes without use of special microscopy systems would be ideal (e.g., the white eye color system by Jasinskiene et al. 1998); however, unless such markers confer a dominant phenotype that can be observed in a wild-type background, they would not be useful for the purpose we describe here. Universal markers using the $3 \mathrm{XP} 3$ promoter have been developed to express fluorescent proteins, which have a dominant expression and can be used in many species, including Tribolium and Drosophila (Berghammer et al. 1999), and in many mosquitoes.

\section{Promoters for fluorescent protein unique markers}

In mosquitoes, only a handful of promoters are used to express fluorescent proteins that are used as transgene markers (rather than tissue-specific markers of expression) (reviewed in Hammond and Nolan 2015), including 3XP3, actin5c, Hr5-IE1, and polyubiquitin. Researchers working with a particular species or group should consider selecting a promoter that is either no longer in use, but has no intrinsic 
deficiencies, or developing a new one. Good native promoter candidates identified by gene expression studies would provide an entrée for candidates. The intention would be to develop and assign a promoter for fluorescent proteins that could be reserved only for autonomous IGFs. While likely impractical for species with many pre-existing transgenic strains covering a range of markers, such a strategy may be implemented at the outset for IGFs developed for species with little prior investigation.

This would provide an identifiable marker to distinguish strains carrying a specific IGF quickly from other conventional transgenic and nontransgenic strains. "If a specific marker can be visualized (e.g., using fluorescent proteins...), all personnel will need to see examples of the modified organisms to avoid confusion with other organisms without the gene drive and provided with appropriate materials, such as vials or cages, for collecting test organisms found outside of their normal area." (NAS Committee, 2016).

The use of such markers would allow even large laboratory populations to be nondestructively and exhaustively screened to ensure that conventional transgenic and wild-type strains are not contaminated. They would also allow nontransgenic and conventional transgenic strains to be examined, before distribution or experiments, to confirm that no IGF is present and to ensure that only material or progeny from these individuals is used. If unique markers were adopted that distinguish different IGFs, contamination among different driving strains would still be unidentified, but it is a risk only to the validity of experimental results and not to containment.

Dedication of a specific marker might be restricted to work within a single laboratory or could consist of a broader agreement among multiple laboratories that are planning IGF activities for a particular species or group. Ideal requirements for such a promoter/marker are (1) it is not currently being used for arthropods that can cross-hybridize; (2) it has a minimum of negative effects on fitness; and (3) it is easily detectable in many, if not all, stages, even in the presence of other commonly used markers. For species in which a marker will be used for field release, persistence of the marker in dead insects will facilitate monitoring.

In the absence of sensitive markers and IGFs that are prone to mutation of the marker, PCR analysis is the only currently practical way to distinguish contamination among similarly marked IGF strains. In this case, PCR assays should be developed that uniquely identify a particular transgene in its specific location for each strain. These can be designed easily based on the flanking genomic DNA sequence and that of the transgene using a unique region that distinguishes it from all the others. Ideally, this will be a multiplex PCR that includes primers that will amplify the empty genomic locus that does not contain the transgene so that it can be used to determine the genotype. Alternatively, this would be facilitated if the research community would agree upon a specific set of primers to amplify portions of the transgene that are found in all constructs of a particular class such as the I-PpoI HEG or a portion of the Cas9 gene. These would permit detection of even a low frequency of a particular class of driving transgenes from populations when subjected to exhaustive analysis.

It may prove difficult for the research community to reach a consensus of markers specific for IGFs in species that they study, but in cases in which it is possible, it will provide advantages that will promote containment and facilitate experimental activities.

\section{Practices to Prevent Contamination}

\section{Routine authentication}

Authentication in regard to transgenic arthropods is the process of confirming salient distinguishing characteristics of a strain. The measures that are appropriate depend on distinctive characteristics of strains being held within the facility and, for example, may consist of confirming the species or subspecies identity, a unique single, or specific combination of alleles, mutation phenotype, or distinguishing molecular markers. These features should distinguish each strain from others being held in the facility.

Inadvertent mixtures of more than one IGF within a strain, even when unique markers have been chosen for IGFs, will rarely be identified when it has occurred. If the IGF markers are not unique, routine molecular analysis using all of the primer sets that are used to authenticate similar strains or phenotype analysis would be necessary. Thus, it is good practice for any laboratory conducting experiments with numerous strains or distributing them to the research community, as a service or part of a collaboration, to routinely perform and document authentication. An example using Anopheles mosquitoes has been provided by the Malaria Research and Reference Reagent Resource Center (MR4, Wilkins et al. 2009). An authentication strategy should consider the stocks within the facility and methods should be developed that recipients will be able to conduct with typical equipment. For laboratories managing transgenic strains, we recommend performing authentication on a regular schedule, and this is even more critical if IGFs are being handled.

Laboratories should develop authentication protocols for strains being held, both to ensure their identity and particularly to detect contamination with an IGF. Ideally, authentication activities should be simple to conduct because they need to be done on a regular schedule that permits recovery of the strain and before critical experiments are conducted with it. The practices include morphological/phenotypic examination, bioassays, and PCR. Aside from containment issues, regular authentication ensures strain purity to protect investments in experiments and ensure that any distributions of insects are in compliance with regulatory or material transfer agreement (MTA) requirements.

\section{Strain segregation}

Strain contamination may be suspected as a result of routine authentication or when unusual phenotypes are noticed during culture or experiments. Strains are assumed to be intact in the absence of such indication of contamination. Many, if not most, insectaries house more than one strain of a particular species or cross-mating group. Ideally, IGFcontaining strains would be housed and manipulated in a separate research space to avoid cross-contamination of wildtype or other non-IGF transgenic strains. While an independent facility dedicated to IGFs would be ideal, an adjoining room within an existing facility or even a dedicated environmental chamber for IGFs should be considered.

Where no separation is possible between IGF-containing strains and other transgenic or wild-type strains, all materials 
held in such a space should be treated as if they contain the IGF. Any transport to another facility would thus require a demonstration that transported life stages are indeed free of the IGF. The authentication procedures described above will assist in this process.

\section{Preventing inadvertent distribution}

Measures must be in place to ensure no IGFs are accidentally distributed. Distribution might occur before a sample from a contaminated colony with a low frequency of the transgene has been detected. If the same marker were being used for both IGFs and non-IGFs and the strain being distributed contained that marker, it would be necessary to analyze the strain using PCR analysis to confirm its purity. Individual analysis of all parents would be necessary either after the progeny had been obtained or by analyzing a tissue sample of parents in a way that allowed them to survive and reproduce (e.g., Pompon and Levashina 2015).

\section{Monitoring insects outside of containment}

Trapping escapees within the insectary should be routine using practical and effective trapping methods that might be available for doing so. It is highly recommended that personnel immediately notify the principal investigator when escapees are found anywhere in the insectary and revisions made to working procedures to prevent further escapes. Monitoring for the presence of escapees in airlocks, corridors, or outside the facility provides an indication of how well containment measures are functioning; data from such efforts should be reported as required by the NIH guidelines. An important consideration concerning the use of monitoring to identify IGF-containing insects both inside and outside a facility is identifying responsible parties for conducting these activities from the outset. Who will be responsible for checking traps at the appropriate frequency? If fluorescence microscopy and PCR are required to identify unique IGFs, the usual responsible party will be the same laboratory that is working with that strain. In this case, conflicts of interest must be carefully managed as such laboratories may have a strong incentive against reporting the presence of escapees. Those charged with approval and regulatory oversight should be cautious to ensure that handling and containment methods do not create situations that actually reduce containment. An example is negative pressure that was discussed above.

\section{Measures to consider for recipients of strains}

Laboratories that receive wild-type or nondriving transgenic strains from laboratories in which IGFs are handled should be provided instructions for verifying that the strain is free of IGFs after receipt. Again, the use of a unique morphological marker for IGFs will facilitate this. Regardless, insectaries that distribute the strains should provide methods for authentication of the strain to the recipients. The use of MTAs should typically involve appropriate biosafety officers at both the shipping and receiving institutions so that they can verify the containment conditions and authentication strategies. Distribution of such strains should be done with particular caution and be validated for safety. If laboratories cannot provide evidence that strains are IGF free, such strains should be handled as if they contained the IGF, with all ap- propriate containment measures. This should be considered by the IBC and other regulatory authorities.

Given that many of the IGFs under study are being developed to control vector-borne diseases in low-income countries, considerations should be made to ensure that appropriate biosafety measures are available in these countries. The Cartagena Protocol on Biosafety was designed, in part, to protect low-income countries against threats to biosafety due to the international movement of genetically modified organisms (Secretariat of the Convention on Biological Diversity 2011). It is in the spirit of this protocol for exporting countries to carry out a comprehensive risk assessment and to ensure that sufficient biosafety measures are in place before the first export of organisms carrying IGFs into low-income countries.

\section{Conclusions}

Previous guidance on facility design and operations at levels 2 and 3 serves as a starting basis for physical containment for developing and testing driving transgenic arthropods such as mosquitoes, with an appreciation that each IGF may need its own special considerations. The increased potential for an increase in frequency and persistence of arthropods containing an IGF present additional concerns that are different from typical transgenic arthropods and arthropods infected with a pathogen.

In principle, low-threshold IGFs that are being investigated in research laboratories present unique opportunities to benefit public health, agriculture, and the environment. To ensure their safe development in arthropods, several measures can be put in place to ensure that inadvertent release, distribution, and contamination by IGFs are prevented. Specific recommendations based on surveys of existing guidance and current knowledge of IGF systems include the following:

- Laboratories that are suitable for handling low-threshold IGFs in arthropods should be managed with additional considerations in mind relative to conventional arthropod-rearing facilities, including strain and facility design, and equally importantly, facility management. Facility modification for this purpose is feasible, particularly if it is already operating at an ACL-2 level.

- Increased arthropod containment measures above typical level 2 measures and active and passive monitoring and buffer zones should be considered, particularly when risk of establishment exists or there are populations in the surroundings. Microbe-specific containment measures are unnecessary unless microbes will be used.

- Where feasible, incorporating a unique dominantacting marker into the IGF construct that allows visual identification of IGF individuals and that distinguishes them from nondriving strains is recommended. Working with unmarked IGFs or a common marker will not allow contamination to be easily recognized, but may be the only recourse in some circumstances. In these cases, definitive PCR-based assays could serve as a substitute. These types of markers are more important in laboratories holding mixtures of non-IGF and IGF strains, particularly when distribution is anticipated.

- Permitting authorities should consider each IGF on a case-by-case basis. The probability that an IGF will 
establish and spread following an accidental release depends on the mechanism of gene drive and the phenotype that is expected to be propagated and the environment into which it may escape.

- The degree of physical containment should be informed by the size of the potential escaping population (dictated, for example, by the total size of the facility), the probability of escape, the likely limited temporal and spatial extent of IGF populations, and an assessment of target and nontarget risks.

- Distribution of nontransgenic and wild-type strains of the same mating group by laboratories that also handle IGFs should be carefully scrutinized to ensure against contamination. If procedures allow for potential contamination, then only individual arthropods that have been exhaustively screened immediately before shipment should be shared and only if it is confirmed that they do not contain an IGF. This will be facilitated by diagnostic PCR and/or unique phenotypic markers.

- Authentication of all strains in the insectary handling IGFs should be conducted routinely, particularly if organisms will be distributed from the facility. Depending on the number of similarly marked strains and the stringency of containment, strain-specific PCR assays may provide the ability to discriminate between strains.

- Routine assessments of management and housing of strains held in insectaries should be performed and modified as conditions warrant.

- Multiple strains should be housed in a way that minimizes the probability of cross-mating and accidental transfer of all life stages and maximizes detection of contamination based on the mating group and markers.

- Containment of all IGF strains, including model species, should be carefully assessed in view of potential legal implications of their unintentional spread, particularly considering international transboundary issues. This is particularly critical for strong IGFs that are expected to spread rapidly.

Implementing some measures addressed here will require building a consensus of practice among those working with a species group, for example, in the selection of a promotermarker combination. Some can be implemented independently by those operating only one laboratory or within a consortium of collaborating laboratories. Early consultations with experts with broader experience, including ethicists, regulators, and IBCs, will help ensure that a range of concerns and mitigations are considered.

Investigators, regulatory authorities, IBCs, and project donors are responsible for ensuring that appropriate procedures are followed for handling of arthropods containing an IGF. These recommendations are intended to supplement current guidance and assist those who face the challenges of making decisions that could be the subject of careful scrutiny by the public and whose consequences may have legal ramifications.

\section{Acknowledgments}

The authors extend their thanks to Hector Quemada for useful comments on the legal aspects of transboundary issues and to Stephanie James for her insights on the content and numerous edits. The authors appreciate the exchanges, edits, and perspectives of Ethan Bier and Anthony James, all of which improved the final recommendations. Clarity and accuracy of the manuscript were enhanced by the excellent reviews of two anonymous reviewers. The findings and conclusions in this report are those of the authors and do not necessarily represent the official position of the Centers for Disease Control and Prevention. Publication costs were graciously provided by Target Malaria which receives core funding from the Bill \& Melinda Gates Foundation and from the Open Philanthropy Project Fund, an advised fund of Silicon Valley Community Foundation.

\section{Author Disclosure Statement}

No competing financial interests exist.

\section{References}

ACME/ASTMH. Arthropod containment guidelines. A project of the American Committee of Medical Entomology and American Society of Tropical Medicine and Hygiene. Vector Borne Zoonotic Dis 2003; 3:61-98.

Akbari OS, Bellen HJ, Bier E, Bullock SL, et al. Safeguarding gene drive experiments in the laboratory. Science 2015; 349 : 927-929.

Akbari OS, Chen C-H, Marshall JM, Huang H. Novel synthetic Medea selfish genetic elements drive population replacement in Drosophila; a theoretical exploration of Medea-dependent population suppression. ACS Synth Biol 2014; 3:915-928.

Benedict M, Scott MJ. Concept and History of Genetic Control. In: Adelman Z, ed. Genetic Control of Malaria and Dengue. New York: Academic Press, 2016:31-46.

Benedict MQ, D'Abbs P, Dobson S, Gottlieb M, et al. Guidance for Contained Field Trials of Vector Mosquitoes Engineered to Contain a Gene Drive System: Recommendations of a Scientific Working Group. Vector Borne Zoonotic Dis 2008; 8:127-166.

Berghammer AJ, Klingler M, Wimmer EA. A universal marker for transgenic insects. Nature 1999; 402:370-371.

Bernardini F, Galizi R, Menichelli M, Papathanos PA, et al. Site-specific genetic engineering of the Anopheles gambiae $\mathrm{Y}$ chromosome. Proc Natl Acad Sci USA 2014; 111:7600-7605.

Bhatt S, Weiss DJ, Cameron E, Bisanzio D, et al. The effect of malaria control on Plasmodium falciparum in Africa between 2000 and 2015. Nature 2015; 526:207-211.

Burt A. Heritable strategies for controlling insect vectors of disease. Philos Trans R Soc Lond B Biol Sci 2014; 369:20130432.

Burt A. Site-specific selfish genes as tools for the control and genetic engineering of natural populations. Proc R Soc Lond B Biol Sci 2003; 270:921.

Cao-Lormeau V-M. (2016) Tropical islands as new hubs for emerging arboviruses. Emerg Infect Dis 2016; 22:913-915.

Department Health and Human Services, Public Health Service. Biosafety in Microbiological and Biomedical Laboratories (5th Edition), Washington, DC, 2016.

Deredec A, Godfray HCJ, Burt A. Requirements for effective malaria control with homing endonuclease genes. Proc Natl Acad Sci USA 2011; 108:E874-E880.

Eckhoff PA, Wenger EA, Godfray HCJ, Burt A. Impact of mosquito gene drive on malaria elimination in a computational model with explicit spatial and temporal dynamics. Proc Natl Acad Sci USA 2017; 114:E255-E264.

Esvelt KM, Smidler AL, Catteruccia F, Church GM. Concerning RNA-guided gene drives for the alteration of wild populations. Elife 2014; 3:e03401. 
Fisher RA. On the dominance ratio. Proc R Soc Edinb 1922; 42: 321-341.

Gabrieli P, Smidler A, Catteruccia F. Engineering the control of mosquito-borne infectious diseases. Genome Biol 2014; 15:1-9.

Galizi R, Doyle LA, Menichelli M, Bernardini F, et al. A synthetic sex ratio distortion system for the control of the human malaria mosquito. Nat Commun 2014; 5:1-8.

Gantz VM, Bier E. The mutagenic chain reaction: A method for converting heterozygous to homozygous mutations. Science 2015; 348:442-444.

Gantz VM, Jasinskiene N, Tatarenkova O, Fazekas A, et al. Highly efficient Cas9-mediated gene drive for population modification of the malaria vector mosquito Anopheles stephensi. Proc Natl Acad Sci USA 2015; 112:E6736-E6743.

Gillies MT, Wilkes TJ. Field experiments with a wind tunnel on the flight speed of some West African mosquitoes (Diptera: Culicidae). Bull Entomol Res 1981; 71:65-70.

Grafton-Cardwell EE, Stelinski LL, Stansly PA. Biology and management of Asian citrus psyllid, vector of the huanglongbing pathogens. Annu Rev Entomol 2013; 58:413-432.

Griffin JT, Hollingsworth TD, Okell LC, Churcher TS, et al. Reducing Plasmodium falciparum malaria transmission in Africa: A model-based evaluation of intervention strategies. PLoS Med 2010; 7: pii: e1000324

Haldane J. A mathematical theory of natural and artificial selection, part V: Selection and mutation. Math Proc Camb Phil Soc 1927; 23:838-844.

Hammond A, Galizi R, Kyrou K, Simoni A. A CRISPR-Cas9 gene drive system targeting female reproduction in the malaria mosquito vector Anopheles gambiae. Nat Biotechnol 2016; 34:78-83.

Hammond AM, Nolan T. Sex-, Tissue- and Stage-Specific Transgene Expression. In: Benedict M, ed. Transgenic Insects: Techniques and Applications. Oxfordshire, United Kingdom: CABI, 2015:29-50.

Hay BA, Chen C-H, Ward CM, Huang H. Engineering the genomes of wild insect populations: Challenges, and opportunities provided by synthetic Medea selfish genetic elements. J Insect Physiol 2010; 56:1402-1413.

Hayes KR, Barry SC. Are there any consistent predictors of invasion success? Biol Invasions 2008; 10:483-506.

Jasinskiene N, Coates CJ, Benedict MQ, Cornel AJ, et al. Stable transformation of the yellow fever mosquito, Aedes aegypti, with the Hermes element from the housefly. Proc Natl Acad Sci USA 1998; 95:3743-3747.

Kidwell MG, Ribeiro JM. Can transposable elements be used to drive disease refractoriness genes into vector populations? Parasitol Today 1992; 8:325-329.

Lefevre T, Gouagna LC, Dabire KR, Elguero E, et al. Evolutionary lability of odour-mediated host preference by the malaria vector Anopheles gambiae. Trop Med Int Health 2009; 14:228-236.

Marshall JM, Akbari OS. Gene drive strategies for population replacement. In: Adelman Z, ed. Genetic Control of Malaria and Dengue. New York: Academic Press, 2016; 169-200.

Marshall JM, Hay BA. Confinement of gene drive systems to local populations: A comparative analysis. J Theor Biol 2012; 294:153-171.

Marshall JM. The effect of gene drive on containment of transgenic mosquitoes. J Theor Biol 2009; 258:250-265.
NAS Committee: Committee on Gene Drive Research in NonHuman Organisms: Recommendations for Responsible Conduct, Board on Life Sciences, Division on Earth and Life Studies, National Academies of Sciences, Engineering, and Medicine. Gene Drives on the Horizon: Advancing Science, Navigating Uncertainty, and Aligning Research with Public Values. Washington, DC: National Academies Press, 2016.

North A, Burt A, Godfray HCJ. Modelling the spatial spread of a homing endonuclease gene in a mosquito population. J Appl Ecol 2013; 50:1216-1225.

Oye KA, Esvelt K, Appleton E, Catteruccia F, et al. Regulating gene drives. Science 2014; 345:626-628.

Pompon J, Levashina EA. A New Role of the Mosquito Complement-like Cascade in Male Fertility in Anopheles gambiae. Plos Biol 2015; 13:e1002255.

Secretariat of the Convention on Biological Diversity. Nagoya-Kuala Lumpur Supplementary Protocol on Liability and Redress to the Cartagena Protocol on Biosafety. Montreal, Canada: Secretariat of the Convention on Biological Diversity 2011.

Sinkins SP, Gould F. Gene drive systems for insect disease vectors. Nat Rev Genet 2006; 7:427-435.

Tabachnick W. Nature, nurture and evolution of intra-species variation in mosquito arbovirus transmission competence. Int J Environ Res Pub Health 2013; 10:249-277.

Tabachnick WJ. Reflections on the Anopheles gambiae genome sequence, transgenic mosquitoes and the prospect for controlling malaria and other vector borne diseases. J Med Entomol 2003; 40:597-606.

TDR/WHO. Progress and Prospects for the Use of Genetically Modified Mosquitoes to Inhibit Disease Transmission. Geneva: World Health Organization, 2010:68.

Ward CM, Su JT, Huang Y, Lloyd AL, et al. Medea selfish genetic elements as tools for altering traits of wild populations: A theoretical analysis. Evol 2010; 65:1149-1162.

Weaver SC, Costa F, Garcia-Blanco MA, Ko AI, et al. Zika Virus: History, Emergence, Biology, and Prospects for Control. Antiviral Res 2016; 230:69-80.

Webber BL, Raghu S, Edwards OR. Is CRISPR-based gene drive a biocontrol silver bullet or global conservation threat. Proc Natl Acad Sci USA 2015; 112:10565-10567.

WHO/TDR, FNIH. Guidance Framework for Testing of Genetically Modified Mosquitoes. Geneva: WHO, 2014.

WHO: World Health Organization. World Malaria Report 2015. Geneva: World Health Organization, 2015.

Wilkins EE, Marcet PL, Sutcliffe AC, Howell PI. Authentication scheme for routine verification of genetically similar laboratory colonies: A trial with Anopheles gambiae. BMC Biotechnol 2009; 9:91.

Windbichler N, Menichelli M, Papathanos PA, Thyme SB, et al. A synthetic homing endonuclease-based gene drive system in the human malaria mosquito. Nature 2011; 473:212-215.

Address correspondence to: Mark Q. Benedict Entomology Branch

Centers for Disease Control and Prevention (CDC) 1600 Clifton Road Atlanta, GA 30333

E-mail: mbenedict@cdc.gov 